\title{
Decreased Response to Growth Hormone after Interruption of Therapy
}

\author{
Yukihiro Hasegawa, Tomonobu Hasegawa, Taiji Aso, Makoto Anzo, Shinobu Kotoh and Yutaka Tsuchiya \\ Division of Endocrinology and Metabolism, Tokyo Metropolitan Kiyose Children's Hospital (YH, TA, MA, SK), \\ Tokyo, Department of Pediatrics, Keio University School of Medicine (TH, YT), Tokyo, Japan
}

\begin{abstract}
The relatively high cost of recombinant human growth hormone (rhGH) has raised the issue of whether intermittent treatment regimens might be efficacious for patients with GH deficiency. Two previous reports have suggested that interruption of GH treatment may avoid the typical waning of response associated with continuous GH therapy and may, therefore, result in an advantageous cost-benefit ratio. We have studied 2 patients with GH deficiency treated with rhGH which was interrupted for one month. The one month of interrupted therapy did not have any beneficial effect and may have caused a loss of potential growth. Although our observations are limited, the data indicate that intermittent GH treatment regimens should not be advocated in the absence of additional investigations.
\end{abstract}

Key words: $\mathrm{GH}, \mathrm{GH}$ therapy, GH deficiency

\section{Introduction}

The relatively high cost of recombinant human growth hormone (rhGH) therapy has raised the question of whether intermittent treatment regimens may be more cost-effective than continuous, daily treatment. Intermittent therapy might be one of the treatment options, especially for patients with a less severe type of $\mathrm{GH}$ deficiency. Recently, Bougneres (1) has reported that the linear growth response after re-initiation of interrupted GH treatment is similar to the response seen during the initial

Received: September 14, 1995

Accepted: January 29, 1997

Correspondence: Dr. Yukihiro Hasegawa, Division of Endocrinology and Metabolism, Tokyo Metropolitan Kiyose Children's Hospital, 1-3-1 Umezono, Kiyose, Tokyo 204, Japan treatment period. In addition, the total incremental height gains for patients treated with intermittent and continuous rhGH regimens were similar. These data suggested that the cost-benefit ratio for intermittent rhGH treatment might be better than for standard continuous therapy.

We report observations for 2 prepubertal children with classical GH deficiency who were treated with continuous rhGH for 1 to 2 years followed by a one month interruption. Our data fail to confirm the results of Bougneres (1).

\section{Case Reports}

Patient 1 was referred to the Endocrinology Clinic at Tokyo Metropolitan Kiyose Children's Hospital at the age of 8 years (yr.) for evaluation of short stature and inadequate growth rate. Physical examination showed a prepubertal boy with a height of $108.6 \mathrm{~cm}$ ( $-3.2 \mathrm{SD}$ below the 
mean for age/sex) (2). The examination was otherwise noncontributory. Growth velocity in the year prior to evaluation was $3.4 \mathrm{~cm} / \mathrm{yr}$. $(-2.9$ $\mathrm{SD}$, Table 1) (2). Subsequent testing revealed TSH deficiency (low T4 and TSH levels) and abnormally low GH and cortisol responses after arginine and insulin stimulation tests; peak serum GH levels were 1.4 and $1.7 \mathrm{ng} / \mathrm{mL}$, respectively (GH RIA, Eiken Chemical Co. Ltd., Tokyo). The patient had suffered head injury during an automobile accident at the age of 5 months. Magnetic resonance imaging (MRI) at the time of endocrine evaluation showed absence of the pituitary stalk with a hypoplastic, anterior pituitary gland. Treatment with $\mathrm{rhGH}, 0.5 \mathrm{U} / \mathrm{kg} /$ week (10 U) divided into $6 \mathrm{sc}$. doses/week, thyroxine $(0.06 \mathrm{mg} /$ day) and hydrocortisone (2.5 $\mathrm{mg} /$ day) was started at the age of $8 \mathrm{yr}$. and 2 months. During the first year of treatment, his height velocity was $10.4 \mathrm{~cm} / \mathrm{yr}$. (+7.5 SD, Table 1). Therapy was interrupted for a period of 31 days beginning at the age of $9 \mathrm{yr}$. and 5 months due to a family relocation. rhGH treatment was then restarted with the same regimen and per $\mathrm{kg}$ dose (adjusted to $12 \mathrm{U} /$ week). Growth velocity over the subsequent year was $5.6 \mathrm{~cm} / \mathrm{yr}$. $(+1.0$ $\mathrm{SD}$, Table 1). The patient remained prepubertal throughout these treatment periods, with normal thyroxine levels and no major acute medical conditions.

Patient 2 was first evaluated at the age of 4 yr. and 6 months for short stature. Physical examination revealed a prepubertal girl with a height of $91.2 \mathrm{~cm}(-3.9 \mathrm{SD})$ and no other significant findings. Past medical history was unremarkable except for short stature. Growth velocity over the previous year was $2.3 \mathrm{~cm} / \mathrm{yr}$. ( -6.4 SD, Table 1). Peak serum GH levels were 3.0 and $2.5 \mathrm{ng} / \mathrm{mL}$ following arginine and insulin stimulation testing, respectively. Basal thyroxine and insulin-stimulated cortisol levels, and a pituitary MRI were normal. After one year of rhGH treatment $(0.5 \mathrm{U} / \mathrm{kg} /$ week divided as a daily sc. dose) her growth velocity was $12.2 \mathrm{~cm} / \mathrm{yr}$. $(+8.1 \mathrm{SD}$, Table 1). At the age of $5 \mathrm{yr}$. and 8 months, one year after initiation of rhGH treatment, therapy was stopped for one month during treatment for pneumonia. rhGH treatment was then restarted with the same regimen and per $\mathrm{kg}$ dose and her growth velocity over the subsequent year was $7.0 \mathrm{~cm} / \mathrm{yr}$. (+1.3 SD, Table 1). The patient remained prepubertal during these periods with normal thyroxine levels and no other acute major medical conditions.

\section{Discussion}

The two prepubertal children with GH deficiency presented here are similar in that they showed an excellent growth response during the first year of rhGH treatment but failed to recover this growth response after one month's interruption and re-initiation of treatment. These results differ from those of Bougneres (1) who found that re-initiation of interrupted $\mathrm{GH}$ treatment resulted in recovery of the initial growth response. This discrepancy may, in part, be due to the relatively short duration of the initial treatment period (12-15 months) for our patients and the short period of interruption (1 month) as

Table 1 Height velocity (HV) in patients-1 and -2

\begin{tabular}{cccc}
\hline \multirow{2}{*}{ Patient } & HV before treatment & \multicolumn{2}{c}{ HV after GH treatment } \\
\cline { 3 - 4 } & & HV before interruption & HV after interruption \\
\hline 1 & $3.4 \mathrm{~cm} /$ year ( $-2.9 \mathrm{SD})$ & $10.4 \mathrm{~cm} /$ year $(+7.5 \mathrm{SD})$ & $5.6 \mathrm{~cm} /$ year $(+1.0 \mathrm{SD})$ \\
2 & $2.3 \mathrm{~cm}$ year $(-6.4 \mathrm{SD})$ & $12.2 \mathrm{~cm} /$ year $(+8.1 \mathrm{SD})$ & $7.0 \mathrm{~cm} /$ year $(+1.3 \mathrm{SD})$ \\
\hline
\end{tabular}

$\mathrm{SD}$; standard deviation. 
compared to $4.5 \pm 0.5 \mathrm{yr}$. and $9.7 \pm 0.1$ months, respectively in the previous report. Our results also differ from studies performed more than 20 years ago in which no waning effect was noted with intermittent GH treatment (3), but the patients in this early report were treated with pituitary hGH which, in retrospect, may have been administered at suboptimal doses.

We conclude that although the possibility of reducing therapeutic costs by using intermittent rhGH regimens is attractive, additional studies will need to be designed to determine whether similar therapeutic efficacy can be achieved for individual patients and, if so, the optimal regimen which should be used. Such studies may also help to clarify the mechanisms of the typical waning effect which occurs during rhGH treatment.

\section{References}

1. Bougneres P. Efficacy of intermittent therapy in growth hormone-deficient children. Eur J Endocrinol 1994; 130: 459-62.

2. Suwa S, Tachibana K, Maesaka H, Tanaka T, Yokoya S. Longitudinal standards for height and height velocity for Japanese children from birth to maturity. Clin Pediatr Endocrinol (Japan) 1992; 1: 5-13.

3. Kirkland RT, Kirkland JL, Librik L, Clayton GW. Results of intermittent human growth hormone therapy in hypopituitary dwarfism. J Clin Endocrinol Metab 1972; 37: 204-11. 